\title{
Survey of bovine norovirus infections from diarrheic calves in South Korea, 2015-2017
}

\author{
Eun-Yong Lee, Hyung-Woo Kang, Ha-Young Kim, Seong-Hee Kim, Boyoun Moon, Byung Jae So, \\ Kyoung-Ki Lee, Yeon-Hee Kim*
}

Animal Disease Diagnostic Division, Animal and Plant Quarantine Agency, Gimcheon 39660, Korea

\begin{abstract}
This study examined complex infections with various enteropathogens and the genetic diversity of bovine norovirus (BNoV) in 932 fecal samples from diarrheic calves in South Korea. Overall, seventeen (1.8\%) of the samples tested positive for BNoV following RT-PCR examination. All BNoV-positive samples were co-infected with other intestinal pathogens, including bovine Rotavirus, Giardia, Cryptosporidium, and Escherichia coli. The genetic diversity of the BNoVs shared high nucleotide identity (98.199.5\%) and amino acid homology (93.5-98.1\%) with genotype 2 BNoV (GIII.2) strains. In conclusion, BNoV infections with GIII genotypes were detected in complex infections of diarrheic calves in South Korea.
\end{abstract}

Keywords: bovine, norovirus, diarrhea, Korea

\section{${ }^{*}$ Corresponding author}

Yeon-Hee Kim

Animal Disease Diagnostic Division, Animal and Plant Quarantine Agency, 177 Hyeoksin 8-ro, Gimcheon-si, Gyeongsangbuk-do 39660, Republic of Korea

Tel: $+82-54-912-0482$

Fax: +82-54-912-0499

E-mail: vetyh@korea.kr

ORCID:

Eun-Yong Lee

https://orcid.org/0000-0002-2388-8757

Hyung-Woo Kang

https://orcid.org/0000-0001-9044-5545

Yeon-Hee Kim

https://orcid.org/0000-0002-8508-7381

Conflict of Interest

The authors declare no conflicts of interest.

Received: November 1, 2018

Revised: January 11, 2019

Accepted: February 18, 2019
Caliciviruses, belonging to the family Caliciviridae, are non-enveloped, single-stranded RNA viruses with positive-sense genomes of 7.4-8.3 kb. Based on genomic organization and genetic analysis [1], the Caliciviridae have been divided into at least four genera: Vesivirus, Lagovirus, Norovirus ( $\mathrm{NoV})$, and Sapovirus as well as a proposed genus, NB-like [2]. Phylogenetically, noroviruses (NoVs) are divided into five genogroups (GI-GV). Human are infected by GI, GII, and GIV NoVs [3], whereas animal NoVs are predominantly categorized as GII (swine), GIII (ruminants), GIV (lions and dogs), and GV (mice) based on the nature of the species infection [4]. NoVs comprise the leading cause of acute nonbacterial gastroenteritis in humans; notably genetically related viruses have been isolated in cattle feces. Currently, five genogroups have been explicated in the genus Norovirus. Depending on genetic homology and phylogenetic relationships, those genogroups have been further subdivided into genotypes. All bovine noroviruses (BNoVs) occupy genogroup III (GIII); thus far, genotype 1 (GIII.1) (Jena/1980/DE) and genotype 2 (GIII.2) (Newbury2/1976/UK) BNoV genomes have been fully sequenced. Among the three open reading frames (ORFs) of the BNoV genome, ORF1 encodes a polyprotein that is processed into six nonstructural proteins (N-terminal protein, NTPase, 3A-like protein, viral genome-linked protein [VPg], 3C-like proteinase, and polymerase). Furthermore, ORF2 encodes a single capsid protein, whereas ORF3 coordinates a minor structural protein [5]. In this study, we examined the presence of BNoVs in Korean calves by performing reverse transcription-polymerase chain reaction (RT-PCR) to determine the genetic diversity of Korean BNoV isolates. During diagnostic examinations of $\mathrm{BNoV}$ infection by RT-PCR, capsid protein sequences were obtained from diarrheic fecal samples, allowing comparisons with previously reported reference strains [6]. A total of 932 bovine diarrheic fecal samples were submitted to APQA from January 2015 to August 2017. Norovirus primers (BNoV-F/BNoV-R), which amplify a 515 bp partial gene of the capsid protein of BNoV GIII and have the following sequences: BNoV-F (5'-CGCTCCATGTTYGCBTGG-3') and BNoV-R (5'-ATCAGCACATGRGGRAACTG-3'), and Maxime RT-PCR premix (Intron, Korea) were used for the detection of noroviruses, as described previously for Korean diarrheic cattle [7]. Positive DNA amplicons (515 nucleotides) were directly sequenced by an ABI Prism 


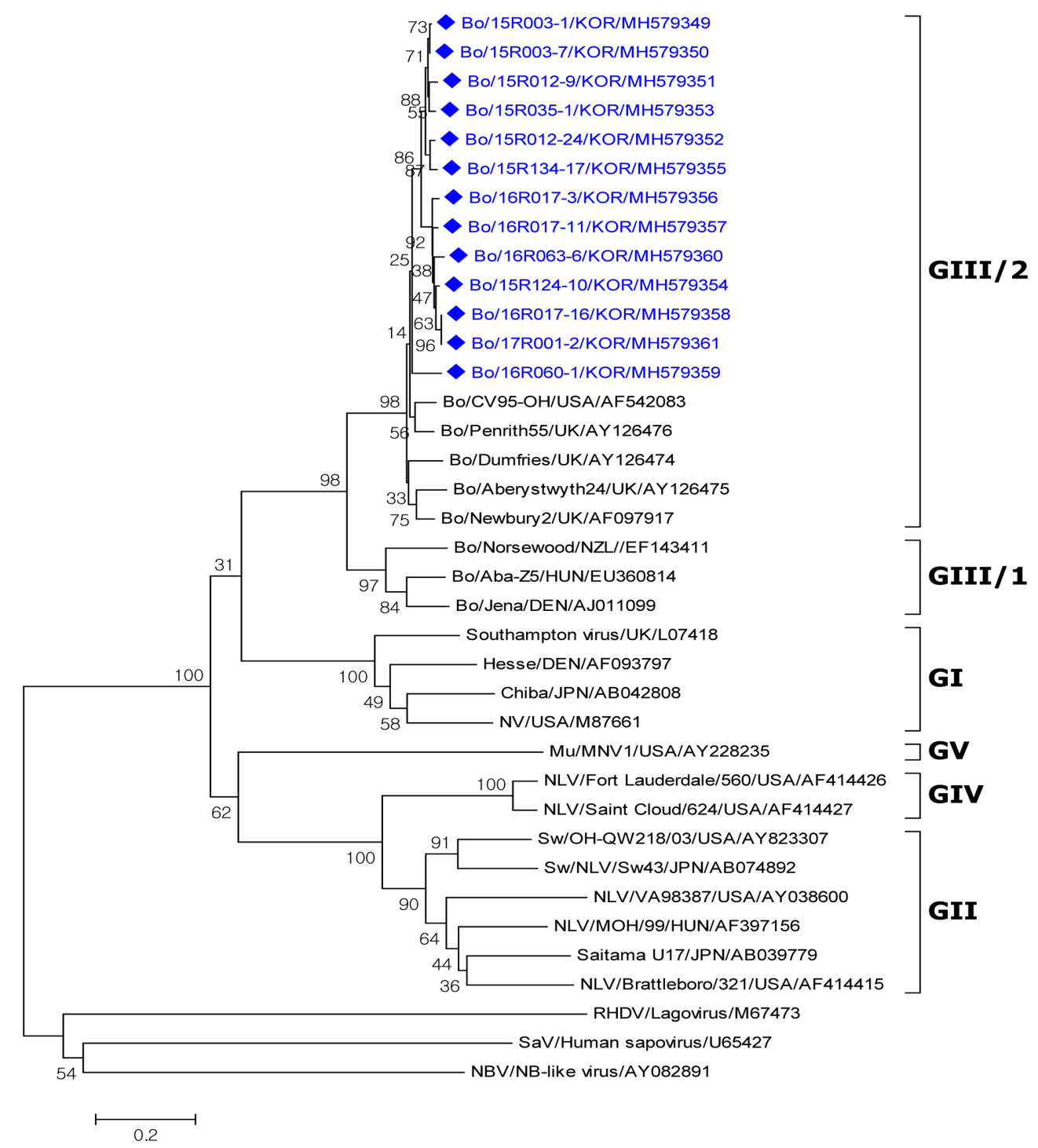

Fig. 1. A phylogenetic tree was constructed on the basis of the partial nucleotide sequence of the capsid protein of caliciviruses by using the maximum likelihood method. GenBank accession numbers (MH579349-MH579361) are registered, except for four identical sequences. The bootstrap value (percent) is given at each node. Viruses for which capsid gene sequences were used for phylogenetic analysis are included. Phylogenetically, noroviruses are divided into five genogroups (GI-GV).

3730XI DNA Sequencer (Applied Biosystems, USA) at the Macrogen Institute (Korea). Comparative analysis of nucleotide sequences was performed by using BioEdit Sequence Editor Version 7.1 software and Molecular Evolutionary Genetic Analysis (MEGA) version 6.0 with bootstrap values calculated from 1000 replicates [8]. The neighbor-joining analysis was used to construct the phylogenetic tree by applying the maximum likelihood method [9]. All diarrheic fecal samples were also tested for the presence of other pathogens, including bovine Rotavirus (BRV), bovine viral diarrhea virus (BVD), bovine Kobuvirus (Kobu), Giardia (GIAR), Cryptosporidium (CRYP), enterohemorrhagic Escherichia coli (EHEC), and Clostridium difficile (CDIFF) [10].

Among the 932 diarrheic fecal samples, 17 (1.8\%) were positive for BNoV. The 17 RT-PCR products were sequenced and analyzed phylogenetically to confirm their nucleotide identity with previously reported $\mathrm{BNoV}$ strains belonging to genotype GIII (GenBank accession numbers: MH579349MH579361). The 17 Korean strains showed 98.1\%-99.5\% nucleotide identity and $93.5 \%-98.1 \%$ amino acid homology with the GIII.2 reference Bo/Newbury2/UK strain (Fig. 1). The ages of the infected calves were between 3 and 40 days. The numbers of BNoV-positive calves at specific ages were as follows: seven at 3-7 days old, five at 8-14 days old, three at 15-21 days old, and two at 22-40 days old. Other bovine enteric pathogens might have important roles in diarrhea symptoms, both clinically and pathologically, because many other intestinal pathogens have been detected in cattle diarrhea [11]. Of the other enteric pathogens tested in this study, BRV was the predominant agent of co-infection with 
Table 1. Summary of the enteric pathogens present in diarrheic fecal specimens obtained from calves

\begin{tabular}{lc}
\hline \hline \multicolumn{1}{c}{ Enteric pathogens } & Number of samples (\%) \\
\hline BNoV plus GIAR & $3(17.6)$ \\
BNoV plus CRYP & $2(11.8)$ \\
BNoV plus BRV & $2(11.8)$ \\
BNoV plus BVDV & $1(5.9)$ \\
BNoV plus Kobu & $1(5.9)$ \\
BNoV plus CDIFF & $1(5.9)$ \\
BNoV plus BRV, Kobu & $2(11.8)$ \\
BNoV plus BRV, EHEC & $1(5.9)$ \\
BNoV plus BVDV, EHEC & $1(5.9)$ \\
BNoV plus CRYP, CDIFF & $1(5.9)$ \\
BNoV plus BRV, Kobu, CRYP & $1(5.9)$ \\
BNoV plus BRV, BVDV, GIAR & $1(5.9)$ \\
Total & 17 \\
\hline
\end{tabular}

GIAR, Giardia; CRYP, Cryptosporidium; BRV, bovine rotavirus; BVDV, bovine viral diarrhea virus; Kobu, bovine kobuvirus; CDIFF, Clostridium difficile; EHEC, Enterohaemorrhagic E. coli.

$\mathrm{BNoV}$ (41.2\%); the remaining pathogens present were GIAR (23.5\%), CRYP (23.5\%), Kobu (23.5\%), BVD (17.6\%), EHEC (11.8\%), and CDIFF (11.8\%). Interestingly, two diarrheic fecal samples tested positive for the presence of four enteric pathogens (Table 1).

Based on comparisons of the amino acid and nucleotide sequences of the capsid protein fragment, $17 \mathrm{BNoV}$ strains belonging to GIII.2 were detected in diarrheic feces of calves in South Korea. Genotypes 1 and 2 were reported in the first Korean domestic report of $\mathrm{BNoV}$ in diarrhea samples that were collected from 2004 to 2005; however, the present results showed that only genotype 2 (GIII.2) was present during sample collection from 2015 to 2017 . Notably, the detection rate of $\mathrm{BNoV}$ infections in diarrheic calves was reported to be $9.3 \%$ during collections from 2004 to 2005 in South Korea [11]. However, no further reports were published until 2017. In other countries, the following BNoV infection rates have been reported: $8.0 \%$ in England [12], $21 \%$ in Italy [13], $31.6 \%$ in Netherlands [14], and $72.0 \%$ in Ohio, USA [2]. $\mathrm{BNoV}$ infections were detected in Turkey at a rate of $1.7 \%$ and were identical to $100 \%$ Italian and Tunisian strains [15]. Based on the RT-PCR analysis, BNoVs were detected in $1.8 \%$ of the fecal specimens examined in this study. These results suggest that the $\mathrm{BNoV}$-positive rate has decreased markedly; moreover, the mean positive rate has decreased since the initial report of $9.3 \%$ in South Korea. Park et al. [11] reported $\mathrm{BNoV}$ infection with only other enteric viruses; however, NoV can participate in a complex infection, which can be shown by simultaneous testing for other intestinal pathogenic microorganisms including bacteria, protozoa and parasites. In this study, we also observed that there was a difference in the BNoV-positive rate according to calf age with the $\mathrm{BNoV}$-positive rate decreasing with age.

This study confirmed the presence of one single genotype of BNoV, the GIII type, and showed that the GIII.2 BNoV was the predominant genotype; results that are consistent with the previously reported $\mathrm{BNoV}$ genogroup and genotype results. Additional pathogenicity studies into single and multiple infections are needed as these BNoVs form endemic infections in diarrheic calves in South Korea.

\section{Acknowledgments}

This project was supported by a grant (N-1543069-201599-03) from the Animal and Plants Quarantine Agency (APQA), Ministry of Agriculture, Food and Rural Affairs (MAFRA), Republic of Korea.

\section{References}

1. Green KY, Ando T, Balayan MS, Berke T, Clarke IN, Estes MK, Matson DO, Nakata S, Neill JD, Studdert MJ, Thiel HJ. Taxonomy of the caliciviruses. J Infect Dis 2000;181 Suppl 2:S322-S330.

2. Smiley JR, Hoet AE, Tråvén M, Tsunemitsu H, Saif LJ. Reverse transcription-PCR assays for detection of bovine enteric caliciviruses (BEC) and analysis of the genetic relationships among BEC and human caliciviruses. J Clin Microbiol 2003;41:3089-3099.

3. Hutson AM, Atmar RL, Estes MK. Norovirus disease: changing epidemiology and host susceptibility factors. Trends Microbiol 2004;12:279-287.

4. Scipioni A, Mauroy A, Vinjé J, Thiry E. Animal noroviruses. Vet J 2008;178:32-45.

5. Mauroy A, Scipioni A, Mathijs E, Ziant D, Daube G, Thiry E. Complete genome sequence of a novel bovine norovirus: evidence for slow genetic evolution in genogroup III genotype 2 noroviruses. J Virol 2012;86:12449-12450.

6. Wise AG, Monroe SS, Hanson LE, Grooms DL, Sockett D, Maes RK. Molecular characterization of noroviruses detected in diarrheic stools of Michigan and Wisconsin dairy calves: circulation of two distinct subgroups. Virus Res 2004;100: 165-177.

7. Wolf S, Williamson WM, Hewitt J, Rivera-Aban M, Lin S, Ball A, Scholes P, Greening GE. Sensitive multiplex realtime reverse transcription-PCR assay for the detection of human and animal noroviruses in clinical and environmental samples. Appl Environ Microbiol 2007;73:5464-5470.

8. Tamura K, Dudley J, Nei M, Kumar S. MEGA4: Molecular Evolutionary Genetics Analysis (MEGA) software version 4.0. Mol Biol Evol 2007;24:1596-1599.

9. Mauroy A, Scipioni A, Mathijs E, Saegerman C, Mast J, Bridger JC, Ziant D, Thys C, Thiry E. Epidemiological study of bovine norovirus infection by RT-PCR and a VLP-based antibody ELISA. Vet Microbiol 2009;137:243-251.

10. Animal and Plant Quarantine Agency. Standard Diagnostic Method of Animal Disease. 4th ed. Animal and Plant Quarantine Agency, Gimcheon, 2017.

11. Park SI, Jeong C, Kim HH, Park SH, Park SJ, Hyun BH, Yang DK, Kim SK, Kang MI, Cho KO. Molecular epidemiology of bovine noroviruses in South Korea. Vet Microbiol 2007;124:125-133.

12. Oliver SL, Dastjerdi AM, Wong S, El-Attar L, Gallimore C, 
36 Eun-Yong Lee, Hyung-Woo Kang, Ha-Young Kim, Seong-Hee Kim, Boyoun Moon, ByungJae So, Kyoung-Ki Lee, Yeon-Hee Kim

Brown DW, Green J, Bridger JC. Molecular characterization of bovine enteric caliciviruses: a distinct third genogroup of noroviruses (Norwalk-like viruses) unlikely to be of risk to humans. J Virol 2003;77:2789-2798.

13. Di Bartolo I, Ponterio E, Monini M, Ruggeri FM. A pilot survey of bovine norovirus in northern Italy. Vet Rec 2011; 169:73.
14. van der Poel WH, van der Heide R, Verschoor F, Gelderblom H, Vinjé J, Koopmans MP. Epidemiology of Norwalk-like virus infections in cattle in The Netherlands. Vet Microbiol 2003;92:297-309.

15. Gulacti IS, Isidan H. Molecular characterization of the bovine noroviruses from diarrheic calves in Turkey. Turk $\mathrm{J}$ Vet Anim Sci 2016;40:428-433. 